\title{
Novel PSEN1 G209A mutation in early-onset Alzheimer dementia supported by structural prediction
}

\author{
Seong Soo A. An ${ }^{1 \dagger}$, Eva Bagyinszky ${ }^{1 \dagger}$, Hye Ryoun Kim², Ju-Won Seok ${ }^{3}$, Hae-Won Shin ${ }^{4}$, SeunOh Bae ${ }^{1}$,
} SangYun $\mathrm{Kim}^{5^{*}}$ and Young Chul Youn ${ }^{4^{*}}$

\begin{abstract}
Background: Three main genes are described as causative genes for early-onset Alzheimer dementia (EOAD): APP, PSEN1 and PSEN2. We describe a woman with EOAD had a novel PSEN1 mutation.

Case presentation: A 54-year-old right-handed woman presented 12-year history of progressive memory decline. She was clinically diagnosed as familial Alzheimer's disease due to a PSEN1 mutation.

One of two daughters also has the same mutation, G209A in the TM-IV of PS1 protein. Her mother had unspecified dementia that began at the age of 40s. PolyPhen2 and SIFT prediction suggested that G209A might be a damaging variant with high scores. 3D modeling revealed that G209A exchange could result significant changes in the PS1 protein.
\end{abstract}

Conclusion: We report a case of EOAD having probable novel PSEN1 (G209A) mutation verified with structural prediction.

Keywords: Alzheimer's disease, Presenilin 1 mutation, Presenilin 1 protein structure, Novel mutation, Structural prediction

\section{Background}

Alzheimer's disease (AD) is the most common form of senile dementia, which could usually occur in patients over 60 years old. AD occurring at younger ages (early onset $\mathrm{AD}$, EOAD) was quite rarely reported (5-10 \% of all AD cases). Genetic background of EOAD was well-defined with three main genes: amyloid precursor protein (APP), presenilin1 (PSEN1) and presenilin2 (PSEN2). PSEN1, located on chromosome 14, was described as the most common causative gene for EOAD, due to the current reports of more than 200 pathogenic mutations (AlzGene, www.alzgene.org). Majority of these mutations were associated with positive family history with autosomal dominant inheritance pattern. Several de novo EOAD cases were also reported, where no additional affected

\footnotetext{
* Correspondence: neuroksy@snu.ac.kr; neudoc@cau.ac.kr

${ }^{\dagger}$ Equal contributors

${ }^{5}$ Department of Neuology, Seoul National University Bundang Hospital and Seoul National University College of Medicine, 300 Gumidong, Bundang-gu, Seongnam-si, Gyeonggi-do 463-707, South Korea

${ }^{4}$ Department of Neurology, Chung-Ang University College of Medicine, Chung-Ang University Hospital, 224-1 Heukseok-dong, Dongjak-Gu, Seoul 06973, South Korea

Full list of author information is available at the end of the article
}

family members was found. The age of disease onset, associated with PSEN1 mutations, could be variable, and the most of the cases occurred at age of onset at $40-50$ years old [1].

In this manuscript, we report a novel PSEN1 G209A mutation in an EOAD patient. This mutation did not exist in any of the AD mutation databases, and it could be a novel causative mutation for EOAD.

\section{Case presentation}

A 54-year-old right-handed woman presented 12-year history of progressive memory decline. She was a university educated woman. At the age of 42 years, her main symptoms were that she forgot names and needed to make notes. She had poorer recall of information than the others at her school of hotel management. In contrast, she was independent in all activities of daily living but her mood was depressed. At initial consultation, when she was 45 years old, general neurological exam was unremarkable. Attention, language and visuospatial function and frontal executive function were normal. Apraxia, dyscalculia and Gerstmann syndrome were not shown. However, Hopkin's verbal learning test showed 
that free-recall score was 21 (5-8-8) and 20 min delayedrecall was 2 . Its recognition score presented that true positive was 8 and false positive was 3 . Delayed recall of Rey figure score was 5.5. Mini-Mental State Examination (MMSE) was 27/30, time orientation 4/5 and three word recall 2/3. Magnetic Resonance Imaging (MRI) scan are shown in Fig. 1a. The upper axial and coronal FLAIR images showed that medial temporal lobe atrophy was not prominent but bilateral parietal atrophy was on. Her $A P O E$ genotype was homozygous for $\varepsilon 3$. Her mother had dementia at the age of $40 \mathrm{~s}$ and died before $60 \mathrm{~s}$. No specific diagnosis was made in her mother. Her father and only brother did not show dementia. She has two daughters, 29 and 26 years old. The initial clinical impression was mild cognitive impairment with depression. However, AD could not be ruled out. She showed progressive deterioration in verbal and visual memory. At the age of 53, her MMSE was $2 / 30$ and clinical dementia rate (CDR) was 3. She was able to respond only to her name and distinguish her daughter from unfamiliar people. She needed personal care. Her brain MRI (Fig. 1a, lower) demonstrated that global cortical atrophy, more prominent in medial temporal and parietal lobes. FDG-PET (fludeoxyglucose- positron emission tomography) of the patient reveals the bilateral temporal, parietal, precuneal and frontal hypometabolism (Fig. 1b). They consent to perform genetic sequencing on her and her 2 daughters. We screened the APP, PSEN1, PSEN2 and PRNP (prion protein) gene.

\section{Genetic analysis of PSEN1 and structural prediction of mutant PSEN 1 protein Methods}

White blood cells (or buffy coat) were separated by centrifugation at $800 \mathrm{~g}$ (30 minutes). Genomic DNA was extracted by GeneAll blood kit (Seoul Korea) as described in the protocol. We performed a polymerase chain reaction (PCR)-based genetic analysis. We used PCR primers for APP exon 16 [2] and 17 [3], PSEN1 exon 4, 5, 6, 7, 8 and $11[4,5]$, PSEN2 exon 4, 5, 6, 7 and 12 [4] and PRNP genes [6]. Single-strand conformation polymorphism (SSCP, Fig. 2a) analysis was performed with the PCR products. Denaturation buffer was added to the amplicons (50:50), which was composed of $95 \%$ formamide, $18 \mathrm{mMol}$

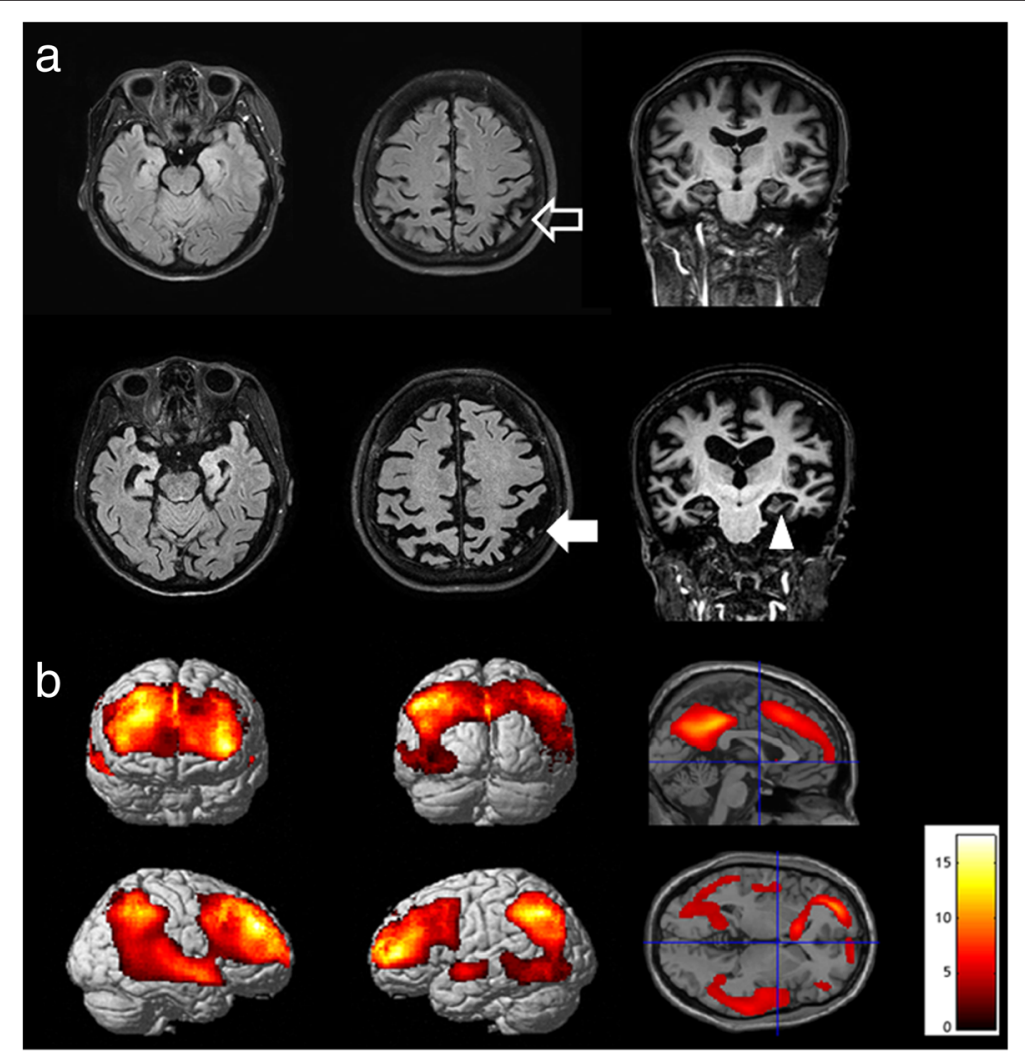

Fig. 1 a Axial and coronal FLAIR images of brain MRI. The upper images at the age of 48 show that bilateral parietal atrophy (empty arrow). The lowers at the age of 54 show that diffuse cortical atrophy and prominent atrophic change in medial temporal (arrow head) and bilateral parietal lobes (filled arrow). b Statistical parametric maps (11 age matched control subjects; $p<0.001$, uncorrected; extent threshold $=50$ voxels) of FDG-PET of the patient, which reveal the bilateral temporal, parietal, precuneal and frontal hypometabolism. The color scale of statistical parametric maps indicates the $Z$ value magnitude, with the lowest value appearing in dark red and the highest value in bright yellow/white 

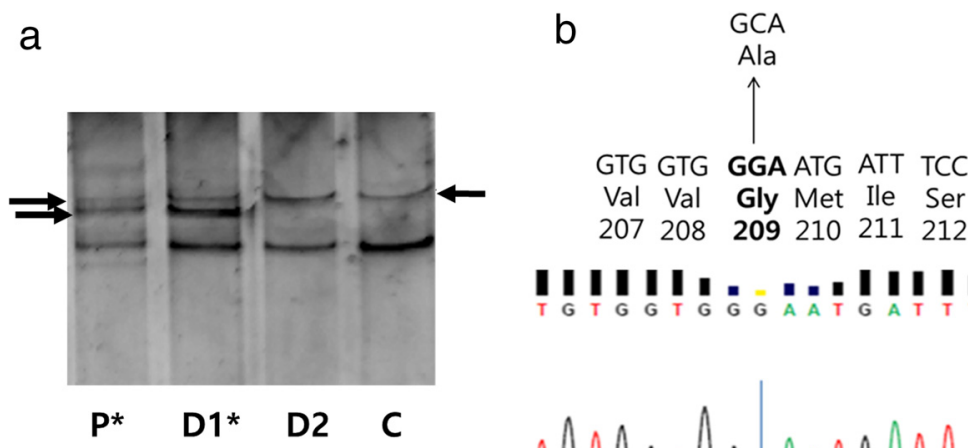

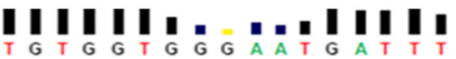

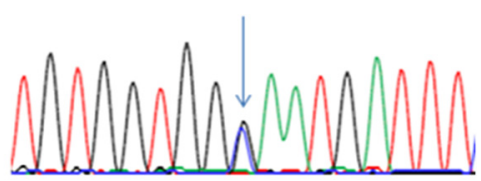

Fig. 2 a SSCP data for PSEN1 G209A. Sample was verified as negative for any mutation in PSEN1 exon7. P*, proband; D1*, affected daughter; D2, unaffected daughter; C, control sample. b Sequencing data of EOAD patient with PSEN1 G209A and location of G209A in PSEN1

EDTA and $0.025 \%$ xylene cyanol, and bromophenol blue. These mixes were incubated on $98{ }^{\circ} \mathrm{C}$ for $10 \mathrm{mi}$ nutes, resulting in the denaturation of double stranded DNA bands [7]. The single stranded DNA bands were separated in non-denaturing polyacrylamide gel electrophoresis (PAGE) (10-12 \%) for 18-21 hours (BioRAD, Seoul, Korea). Depending on the mutations, single stranded DNAs could have different mobility in the gel. SYBR Gold staining (Invitrogen, USA) was used for staining DNA bands, which visualized under UV light. To confirm and identify the mutation, sequencing was performed for all PCR products at both directions by the BioNeer Inc. (Dajeon, Korea). Prior to sequencing, we purified the PCR products by GeneAll PCR protocol kit (Seoul, Korea), as described in the protocol. For the sequencing reactions, Big Dye Terminator Cyclic sequencing was performed on ABI 3730XL DNA Analyzer (http:// eng.bioneer.com/home.aspx, Bioneer Inc., Dajeon, Korea) was used (Fig. $2 \mathrm{~b}$ and c). We aligned the sequenceing results by NCBI Blast (http://blast.ncbi.nlm.nih.gov/Blast.cgi), and the chromatograms were analyzed by DNA BASER (http://www.dnabaser.com) software. Mutations and sequence variants were identified by using the NCBI Gene (http://www.ncbi.nlm.nih.gov/gene) and UniProt (http://www.uniprot.org) databases.

Mutations were analyzed by an online software, the Polymorphism Phenotyping v2 (PolyPhen 2, http:// genetics.bwh.harvard.edu/pph2/), which could make in silico predictions on the putative damaging properties of mutations. This software made a comparison on the wild-type and mutant alleles of proteins by homology searching and multiple sequence alignment. Identityand structure-based searches, such as accessible surface area and hydrophobicity features, were also performed. These predictions might define the potential role of amino acid exchanges in the proteins. Three categorized of mutations could be available: probably, possibly damaging or benign variants. PolyPhen2 could also provide a multiple sequence alignment, by comparing the homologous sequences from different vertebrate and non-vertebrate species. Two types of datasets could be available: the HumDiv and HumVar scores. HumDiv should be used for the prediction of putative pathogenic nature of rare alleles. In HumDiv scores, the alleles with mildly damaging properties should be categorized as possibly damaging. HumVar should be used in case of diagnosis of Mendelian disorders. Here, the highly damaging phenotypes should be distinguished from the alleles with less damaging phenotypes [8].

Sorting Intolerant from Tolerant or SIFT algorithm (http://sift.jcvi.org/) is an online software, which estimates the potential effects of missense mutations substitution on the protein function. SIFT uses several protein databases, including SWISS-PROT, SWISSPROT/TrEMBL, or protein databases of NCBI, and calculates the possibility on the damaging nature of mutations by the comparison of the mutant and normal proteins. It scores the amino acid substitutions, and under and over the score of 0.05, mutations could be defined as deleterious or tolerated, respectively diction [9].

3D structures of PS1 with mutation(s) were analyzed by an online software, the Raptor X (http://raptorx.uchicago.edu/, Fig. 3). Server of protein structure prediction used the full amino acid sequence of PS1 (1-467 amino acids). Discovery Studio 3.5 Visualizer from Accelrys was used to display the images after superimposition [10].

\section{Results}

Alternative mobility was found in the SSCP (Fig. 2a) for exon 7 of PSEN1 gene. Sequencing revealed a $\mathrm{G} \rightarrow \mathrm{C}$ exchange at codon 209, resulting in a glycine (GGA) $\rightarrow$ Alanine (GCA) exchange (Fig. 2b and c). This mutation 

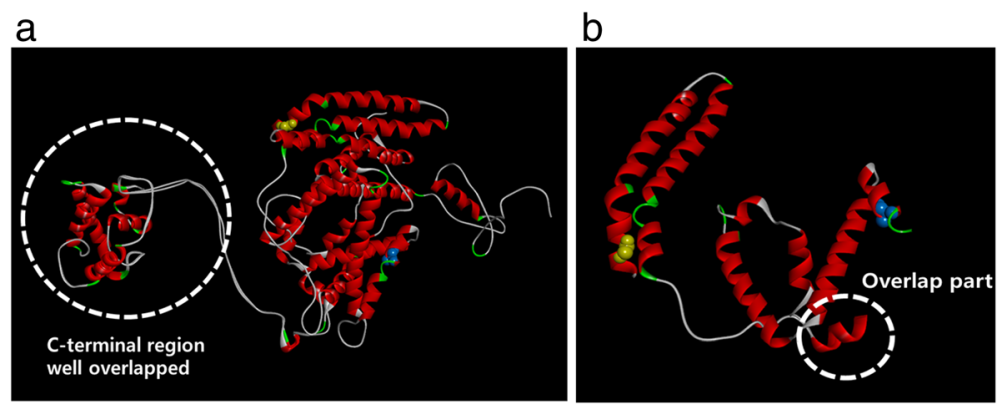

Fig. 3 3D model modeling for PS1 G209A. Differences of normal and mutant PSEN1 have been highlighted in the black circle, and glycine was labeled with yellow, while alanine was labeled with yellow. The C-terminal pert of normal and abnormal proteins were overlapped, while significant differences could be seen in the N-terminal part, suggesting that the mutation could result significant conformational changes in the TM structure of PSEN1. a Differences between normal PSEN1 and PSEN1 G209A, focused on all over the protein. $\mathbf{b}$ Differences between normal and mutant PSEN1, focused on the TM-IV region.

was not found in neither in the AD and FTD mutation database nor in the Alzgene database, which suggested that it might be a novel mutation in PSEN1. Mutation was checked in the Exome Aggregation Consortium (ExAC) database, which screened 60,706 unrelated individuals for the different disease causing genes (http://exac.broadinstitute.org/). PSEN1 G209A was not found in this database.

PolyPhen-2 scores were high, both HumDiv and HumVar was 1.000 (sensitivity: 0.00; specificity: 1.00 at both scores) suggesting G209A as a probably damaging variant. Multiple sequence alignment suggested that glycine 209 might be a conservative residue among vertebrates. Several mammalian (such as dogs, pigs) and non-mammalian (such as chicken, frog, zebrafish) showed glycine at the same position in their presenilin or presenilin-like proteins. SIFT prediction also confirmed the probably damaging nature of mutation, with the score of 0 .

3D prediction (Fig. 3) revealed significant differences in the N-terminal region of normal PS1 protein. The cterminal region of normal PS1 and PS1 with G209A were overlapped. These findings suggest that even glycine and alanine are both small sized, hydrophobic amino acids, but one extra $-\mathrm{CH} 3$ group on alanine could result significant differences in the protein structure. Since alanine is more hydrophobic than glycine, it could result extra stress inside the helix.

\section{Discussion}

We reported a novel mutation in PSEN1, the G209A in a female patient in her early 50 s. Even though we could not find any affected family members, one of her daughters is a mutation carrier and deceased mother had an undiagnosed case of dementia. Our finding suggested that this mutation could be a novel probably pathogenic variant, involved in EOAD.

Glycine 209 is located in the transmembrane -IV region of PS1 protein, and it was suggested as a conservative residue. For codon 209, three additional mutations were found, G209R, G209E and G209V (Table 1), which also suggested that Glycine 209 might be an important residue in PS1. G209R was found in a Japanese family. The proband was a male patient, the disease progression started at the age of 46. Additional affected family

Table 1 Comparison of G209R, G209E, G209V and G209A

\begin{tabular}{|c|c|c|c|c|}
\hline Mutation & Gly209Arg & Gly209Glu & Gly209Val & Gly209Ala \\
\hline Age of onset & $46-53$ years & unknown & 30 - 48 years & 54 years \\
\hline Age of death & unknown & unknown & unknown & Surviving \\
\hline Family history & positive & $\begin{array}{l}\text { Unknown } \\
\text { (detected in } 1 \text { patient) }\end{array}$ & positive & $\begin{array}{l}\text { Unknown (since her mother had } \\
\text { undiagnosed early-onset dementia) }\end{array}$ \\
\hline Clinical phenotype & $\begin{array}{l}\text { Rapid progressive dementia } \\
\text { characterized by memory } \\
\text { impairment, amnestic aphasia, } \\
\text { disorientation and personality } \\
\text { change, but lacking parietal } \\
\text { focal symptoms such as } \\
\text { apraxia or agnosia }\end{array}$ & unknown & $\begin{array}{l}\text { AD with myoclonus, seizures, } \\
\text { language loss, aphasia }\end{array}$ & $\begin{array}{l}\text { Mild cognitive impairment with } \\
\text { depression, followed by progressive } \\
\text { deterioration in verbal and visual } \\
\text { memory }\end{array}$ \\
\hline Reference & Sugiyama et al. 1999 [11] & Rogaeva et al. 2001 [14] & $\begin{array}{l}\text { Poorkaj et al. } 1998 \text { [12] } \\
\text { Larner et al. } 2006 \text { [13] }\end{array}$ & \\
\hline
\end{tabular}


members were found, since his sister and mother also showed clinical symptoms. All of these patients showed rapid progression of dementia, with memory impairment, amnesia, aphasia, disorientation and personality changes. Parietal focal symptoms such as apraxia or agnosia were not apparent in the patients [11]. G209V was found in a German family, where the age of onset ranged between 30-48 years. Clinical symptoms included myoclonus, seizures, language loss and apraxia and agnosia [12, 13]. G209E was reported in one AD patient by Rogaeva. No detailed information was found on the disease age of onset or clinical phenotype of disease, associated with G209E [14].

In silico modeling and the previously described mutations for G209 could support that PSEN1 G209A could be a novel causative mutation for AD. Glycine and alanine are similar, both of them are small sized hydrophobic amino acids. 3D prediction suggested that in this mutation, a single extra $-\mathrm{CH} 3$ group could result disturbances inside the PS1 protein. Since alanine is more hydrophobic than glycine, it could enhance the stress inside the PS1 TM domain or between PS1 and its binding partners.

As conclusion, we suggest that G209A can be a novel, probably pathogenic variant in PSEN1. Guerrio et al. published an algorithm for the novel mutations in PSEN1 and PSEN2. Several criteria were analyzed: such as whether the mutation is segregates with the disorder segregation; whether there are additional affected family members; and whether additional mutations were described for the screened residue [15]. PSEN1 G209A was not found in the ExAC database, which could be a strong evidence on its pathogenic nature. The additional three, probably pathogenic mutations for the glycine at position 209 (G209R, G209V and G209E) can also enhance the importance of residue 209 in PSEN1 and the probably damaging nature of G209A. However, the family history of patient could not be well-defined. Her mother was a dementia patient, but it remained undiagnosed, so it is unclear whether she is the carrier of mutation. One of her daughter carried the mutation, who might be in the pre-symptomatic stage of disorder. Online predictions were suggested that PSEN1 G209A mutation could be a probably deleterious variant. Additional studies are needed on for this variant since its pathogenic nature can't rely on the in silico predictions only.

\section{Conclusions}

We suggest that G209A can be a novel, probably pathogenic variant in PSEN1. Combination of clinical-, association studies, prediction- and 3D modeling software can enhance the estimation on the pathogenic nature of novel variants.

\section{Consent}

Written informed consent was obtained from the legal guardian of patient (her daughter) for publication of this Case report and any accompanying images. They also consent to perform genetic sequencing on their mother and themselves. A copy of the written consent is available for review by the Editor of this journal.

\section{Abbreviations}

AD: Alzheimer's disease; EOAD: Early-onset Alzheimer's disease; MMSE: Minimental state examination; MRI: Magnetic resonance imaging; FDGPET: Fludeoxyglucose- positron emission tomography; TM-IV: Transmembrane segment IV; PS1: Presenilin-1; APP: Amyloid precursor protein; PSEN1: Presenilin 1; PSEN2: Presenilin 2; PRNP: Prion protein; PCR: Polymerase chain reaction; PAGE: Polyacrylamide gel electrophoresis; PolyPhen2: Polymorphism phenotyping V2; SIFT: Sorting intolerant from tolerant.

\section{Competing interests}

The authors declare that they have no competing of interests.

\section{Authors' contributions}

EB and SSAn: drafting the manuscript for content and doing genetic analysis. HRK: preparing the samples and revising the manuscript. JS: verifying the FDG-PET results. HS: interpreting the clinical data and revising the manuscript. SB: predicting presenilin 1 protein structure. SYK: analysis or interpretation of data, doing study supervision, obtaining funding. YCY: drafting/revising the manuscript and interpretation of clinical data. All authors read and approved the final manuscript.

\section{Acknowledgements}

This research was supported by a grant of the Korea Health Technology R\&D Project through the Korea Health Industry Development Institute (KHIDI), funded by the Ministry of Health \& Welfare, Republic of Korea (HI14C3331)

Dr. An SS reports no disclosure.

Dr. Bagyinszky E reports no disclosure.

Dr. Kim HR reports no disclosure.

Dr. Seok J reports no disclosure.

Dr. Shin $\mathrm{H}$ reports no disclosure.

Mr. Bae S reports no disclosure.

Dr. Kim $S$ receives a grant of the Korea Health Technology R\&D Project through the Korea Health Industry Development Institute (KHIDI), funded by the Ministry of Health \& Welfare, Republic of Korea (HI14C3331).

Dr. Youn YC reports no disclosure.

\section{Author details}

${ }^{1}$ College of Bionano Technology, Gachon Bionano Research Institute, Gachon University, Seongnam-si, South Korea. ${ }^{2}$ Department of Laboratory Medicine, Chung-Ang University College of Medicine, Seoul, South Korea. ${ }^{3}$ Department of Nuclear Medicine, Chung-Ang University College of Medicine, Seoul, South Korea. ${ }^{4}$ Department of Neurology, Chung-Ang University College of Medicine, Chung-Ang University Hospital, 224-1 Heukseok-dong, Dongjak-Gu, Seoul 06973, South Korea. ${ }^{5}$ Department of Neuology, Seoul National University Bundang Hospital and Seoul National University College of Medicine, 300 Gumidong, Bundang-gu, Seongnam-si, Gyeonggi-do 463-707, South Korea.

Received: 30 September 2015 Accepted: 9 May 2016 Published online: 20 May 2016

\section{References}

1. Bagyinszky E, Youn YC, An SS, Kim S. The genetics of Alzheimer's disease. Clin Interv Aging. 2014;9:535-51.

2. Tanzi RE, Vaula G, Romano DM, Mortilla M, Huang TL, Tupler RG, Wasco W, Hyman BT, Haines JL, Jenkins BJ et al. Assessment of amyloid beta-protein precursor gene mutations in a large set of familial and sporadic Alzheimer disease cases. Am J Hum Genet. 1992;51 (2):273-82.

3. Schellenberg GD, Pericak-Vance MA, Wijsman EM, Moore DK, Gaskell Jr PC, Yamaoka LA, Bebout JL, Anderson L, Welsh KA, Clark CM et al. Linkage 
analysis of familial Alzheimer disease, using chromosome 21 markers. Am Hum Genet. 1991;48(3):563-83.

4. Cruts $M$, van Duijn $C M$, Backhovens $H$, Van den Broeck $M$, Wehnert $A$, Serneels S, Sherrington R, Hutton M, Hardy J, St George-Hyslop PH et al. Estimation of the genetic contribution of presenilin-1 and -2 mutations in a population-based study of presenile Alzheimer disease. Hum Mol Genet. 1998;7(1):43-51.

5. Kamimura K, Tanahashi H, Yamanaka H, Takahashi K, Asada T, Tabira T. Familial Alzheimer's disease genes in Japanese. J Neurol Sci. 1998; 160(1):76-81.

6. Jeong BH, Ju WK, Huh K, Lee EA, Choi IS, Im JH, Choi EK, Kim YS. Molecular analysis of prion protein gene (PRNP) in Korean patients with CreutzfeldtJakob disease. J Korean Med Sci. 1998;13(3):234-40.

7. Hayashi K. PCR-SSCP: a simple and sensitive method for detection of mutations in the genomic DNA. PCR Methods Appl. 1991;1(1):34-8.

8. Adzhubei IA, Schmidt S, Peshkin L, Ramensky VE, Gerasimova A, Bork P, Kondrashov AS, Sunyaev SR. A method and server for predicting damaging missense mutations. Nat Methods. 2010;7(4):248-9.

9. Ng PC, Henikoff S. SIFT: Predicting amino acid changes that affect protein function. Nucleic Acids Res. 2003;31(13):3812-4.

10. Kallberg M, Wang H, Wang S, Peng J, Wang Z, Lu H, Xu J. Template-based protein structure modeling using the RaptorX web server. Nat Protoc. 2012; 7(8):1511-22.

11. Sugiyama N, Suzuki K, Matsumura T, Kawanishi C, Onishi H, Yamada Y, Iseki E, Kosaka K. A novel missense mutation (G209R) in exon 8 of the presenilin 1 gene in a Japanese family with presenile familial Alzheimer's disease. Mutation in brief no. 254. Online. Hum Mutat. 1999;14(1):90.

12. Poorkaj P, Sharma V, Anderson L, Nemens E, Alonso ME, Orr H, White J, Heston L, Bird TD, Schellenberg GD. Missense mutations in the chromosome 14 familial Alzheimer's disease presenilin 1 gene. Hum Mutat. 1998;11(3):216-21.

13. Larner AJ, Doran M. Clinical phenotypic heterogeneity of Alzheimer's disease associated with mutations of the presenilin-1 gene. J Neurol. 2006; 253(2):139-58

14. Rogaeva EA, Fafel KC, Song YQ, Medeiros H, Sato C, Liang Y, Richard E, Rogaev El, Frommelt P, Sadovnick AD et al. Screening for PS1 mutations in a referral-based series of AD cases: 21 novel mutations. Neurology. 2001 57(4):621-5.

15. Guerreiro RJ, Baquero M, Blesa R, Boada M, Bras JM, Bullido MJ, Calado A, Crook R, Ferreira C, Frank A et al. Genetic screening of Alzheimer's disease genes in Iberian and African samples yields novel mutations in presenilins and APP. Neurobiol Aging. 2010;31(5):725-31.

\section{Submit your next manuscript to BioMed Central and we will help you at every step:}

- We accept pre-submission inquiries

- Our selector tool helps you to find the most relevant journal

- We provide round the clock customer support

- Convenient online submission

- Thorough peer review

- Inclusion in PubMed and all major indexing services

- Maximum visibility for your research

Submit your manuscript at www.biomedcentral.com/submit

C Biomed Central 